\title{
Tecnologías Interactivas en la Zona Arqueológica de Teotihuacán
}

\author{
Interactive Technologies in the Teotihuacan Archeologic Zone \\ Marcos Espítu Vargas ${ }^{a}$, David Martínez Estrada $^{b}$, Mayra Anaid Valerio Nolasco $^{c}$
}

\begin{abstract}
:
Currently, technologies are being applied to different fields of research, among them is learning, within this area, technologies seek to understand how it is that human beings learn and how they are more likely to retain the information; All these advances have been applied in places such as museums, these are referred to as "cultural spaces dedicated to the collection of pieces of heritage value for a region", th is coupled with the technological innovations that have emerged in the area of learning.Spaces called "Interactive Museums" with the support of technologies are emerging, seeking to provide information in a more attractive way for visitors, currently there are several cases and every day, due to the success generated in recent decades, this because of the great value it has as a world heritage site and the lack of information and care suffered by the Area.
\end{abstract}

Keywords:

Interactive technologies, learning, interactive museums, Teotihuacán archeological zone

\section{Resumen:}

En la actualidad las tecnologías están siendo aplicadas a distintos campos de la investigación, entre ellos se encuentra el aprendizaje, dentro de esta área, las tecnologías buscan comprender el cómo es que los seres humanos aprenden y de qué manera es más probable que se retenga la información; Todos estos avances se han aplicado en lugares como son los museos, denominados como "espacios culturales dedicados a la recolección de piezas de valor patrimonial para una región", esto aunado a las innovaciones tecnológicas que han su rgido en el área del aprendizaje. Surgen espacios denominados "Museos interactivos" con apoyo de las tecnologías buscan brindar la información de forma más atractiva para los visitantes, actualmente están surgiendo diversos casos y cada día más, debido al éxito generado en las últimas décadas, esto por el gran valor que tiene como Patrimonio de la Humanidad.

\section{Palabras Clave:}

Tecnologías interactivas, aprendizaje, museos interactivos, Zona arqueológica de Teotihuacán

\section{Introducción}

El uso de tecnologías en algunos sitios culturales se ha vuelto una tendencia que llega a diferir en los beneficios que esto conlleva, sin embargo, la principal razón es lograr un mejor aprovechamiento del lugar para el aprendizaje, así mismo potencializar el nivel cultural y educativo. Los museos usualmente son visitados como mandato de una tarea escolar o como recreación, esto con la finalidad de obtener nuevos conocimientos y convirtiendo los museos como una herramienta dentro del proceso de enseñanza aprendizaje; sin embargo, en la nueva era de las tecnologías, dichos lugares deben modificar sus procesos de enseñanza ya que: "Este tipo de espacios son terreno propicio para impulsar una educación transformadora, y se considera que mediante el uso racional de herramientas digitales se puede

\footnotetext{
a Alumno de octavo semestre del programa educativo de Turismo de la ESTi (Escuela Superior de Tizayuca), Universidad Autónoma del Estado de Hidalgo, https://orcid.org/0000-0002-9362-5573, email: es356867@uaeh.edu.mx

b Alumno de octavo semestre del programa educativo de Turismo de la ESTi (Escuela Superior de Tizayuca), Universidad Autónoma del Estado de Hidalgo, https://orcid.org/0000-0002-0382-0309, email: ma358392@uaeh.edu.mx

c Mtra. Ciencias Sociales, docente de tiempo completo del programa educativo de Turismo de la ESTi (Escuela Superior de Tizayuca), Universidad Autónoma del Estado de Hidalgo, https://orcid.org/0000-0003-2155-1250, email: mayra_valerio@uaeh.edu.mx
} 
desencadenar un proceso de reflexión y resignificación de la experiencia que ofrecen los recintos" (Bedolla, 2015). Una razón para emplear estas tecnologías es que sean un apoyo y que promuevan los procesos de aprendizaje de manera innovadora y atractiva para el visitante por ejemplo dentro de un contexto más real y ya establecido, es el "Museo Interactivo de Economía" MIDE, ubicado en la Ciudad de México, el cual cuenta con medios interactivos que son de apoyo durante su recorrido.

La zona arqueológica de Teotihuacán se ubica en el valle de Teotihuacán en el Estado de México a 62.6 kilómetros del Zócalo de la Ciudad de México, es una de las principales Zonas Arqueológicas a nivel Nacional y la primera en tener mayor número de visitas dentro del turismo nacional (Ortega, 2017), esto gracias a las visitas por parte de las instituciones educativas, dentro de la zona se encuentran las Pirámides del Sol, de la Luna y la Calzada de los Muertos, además de otras tres Zonas Arqueológicas ubicadas en el mismo lugar.

\section{Tecnologías aplicadas al aprendizaje}

De acuerdo con el Departamento de Pedagogía de la Universidad de Girona, España. La interactividad "describe la relación de comunicación entre un usuario y una máquina, estableciendo un proceso de actuación participativa-comunicativa con los materiales" (Meritxell, 2002). Acorde con el concepto brindado por la Universidad de Girona se entiende por interactividad a la interacción que se da entre los seres humanos con la máquina, por ende, la actuación hombre-hombre no se define como interactividad, sino como relación.

Se entiende que el aprendizaje "Es aquel proceso mediante el cual se obtienen los conocimientos, habilidades y competen cias de algún tema en específico" (Garcia-Allen, 2019). Para llegar a esto en la mayoría de los casos se necesitan de dos elementos, el estudio y la práctica de dicho tema. Este concepto es demasiado amplio, ya que existen diferentes vertientes y puntos de vista, pero se puede conceptualizar en que el aprendizaje puede ser intelectual, procedimental o incluso físico.

Existen diferentes formas de definir y diferenciar el aprendizaje, ya que cada persona tiene su manera de adquirir conocimientos y hay diversas maneras o técnicas para la adquisición del aprendizaje. Dentro de los tipos de aprendizajes más comunes son (Garcia-Allen, 2019):

- Aprendizaje Implícito: Este en particular se distingue porque la persona no es consiente sobre que se aprende, teniendo como resultado la ejecución de lo aprendido automáticamente, ejemplos tan básicos como el aprender a hablar o caminar, también conocido como "conducta motora".

- Aprendizaje Explícito: Se caracteriza porque el aprendiz es consciente y tiene la intención de aprender, este tipo de aprendizaje exige de la atención completa de la persona.

- Aprendizaje Asociativo: Se caracteriza por la relación entre dos estímulos, o en algún caso de un estímulo y un comportamiento.

- Aprendizaje Asociativo: En este la persona realiza una selección de información, la organiza y establece ciertas relaciones entre su conocimiento que ya ha adquirido, es decir, la selección y aprovechamiento de la información nueva.

- Aprendizaje Cooperativo: Normalmente se necesita de estar en grupo, requiere de la integración de varias personas que permita el intercambio de pensamientos, la distribución de funciones y que rol desempeña cada uno.

- Aprendizaje Emocional: Funciona para conocer y gestionar las emociones de cada uno de la manera más eficiente posible, esto es de gran beneficio ya que contribuye al bienestar personal, las relaciones humanas y el desarrollo personal.

- Aprendizaje Observacional:Se caracteriza por la integración de dos sujetos en el que uno se encuentra en la participación de modelo, y el otro observa las conductas y aprende.

- Aprendizaje Experiencial: Es aquel que como su nombre lo dice se aprende de las experiencias, en donde se obtiene a base de la práctica y en muchos casos a consecuencia de los errores, teniendo en cuenta que cada individuo obtiene de diferente manera cada vivencia.

- Aprendizaje por Descubrimiento: También llamado aprendizaje activo, en el que la persona se concentra en descubrir, relacionar y ordenar los conocimientos para adaptarlos a su esquema cognitivo.

- Aprendizaje Memorístico: Tiene la característica de memorizar cada concepto en vez de tratar de aprenderlo, esto provoca que se creen acciones repetitivas y mecánicas.

De acuerdo con lo anterior se puede describir que las "Nuevas Tecnologías" propician al aprendizaje implícito, explicito, asociativo, observacional principalmente, esto debido a las nuevas formas de aprender mediante diferentes elementos externos.

\section{1 ¿Cuáles son las tecnologías interactivas más atractivas y usadas en la actualidad?}


De acuerdo con la premisa, de que es necesario el uso de internet y dispositivos que acepte la interacción, se consideran como propuestas las siguientes herramientas que permitan la interactividad según Vallejo (Vallejo, 2015):

- Redes Sociales: Cada día es más utilizada dentro de los museos ya que funciona como un canal de comunicación e interacción con el visitante, lo que permite brindar la información de exposiciones, actividades, horarios, entre otros.

- Wi-Fi: Facilita la interacción tecnológica a través de la conexión a la red del museo. El usuario podrá visualizar la información de las obras.

- Apps de museo: Algunos museos la han desarrollado, en ellas se ofrece agendas, rutas 0 actividades ofrecidas por el mismo.

- Código QR: Actualmente una de las más usadas gracias a la diversidad de usos que presenta, va desde la descarga de información de las obras o exposiciones hasta prácticas más interactivas.

- Podcast: Son dispuestos por parte del museo, el usuario lo descarga al teléfono y lo puede reproducir durante el recorrido, esto sustituye a las audioguías.

- Colecciones Digitales: Son los recorridos virtuales que están dando en los museos, en algunos casos son de carácter contemplativo, pero actualmente se están desarrollando dispositivos que permiten la interacción y modificación de las obras que se encuentran dentro del recorrido virtual.

- Realidad Aumentada: Permite la interacción con la obra, ya, enfocándola, mostrará la información detallada en el dispositivo.

- Obras de Arte Digitales: Un nuevo modo de creación artística que está tomando participación en los museos más modernos.

- Impresoras 3d: Museos como "British Museum" ha puesto a disposición la descarga gratuita de 14 piezas de su colección para poder ser impresas en 3d.

- Gafas Tecnológicas: Mediante las gafas se ofrece información, las gafas detectan el movimiento de los ojos para poder dar la información determinada, una desventaja es que esta tecnología aun no es muy comercial y no ha llegado a los museos de México.
- Mesas Interactivas: Funcionan con bolígrafos que en su interior tienen una memoria USB lo que permite la interacción con las obras presentadas en la mesa.

\section{El aprendizaje en los museos.}

En una perspectiva tradicional, "el museo como institución es, esencialmente, un espacio para la vivencia de sensaciones estéticas, para mostrar objetos, recoger evidencias científicas, organizar investigaciones y construir una plataforma de conocimiento a partir de colecciones de piezas al entorno de un determinado objeto de conocimiento y musealización" (Cardona, Xavier, \& Campillo, 2009).

Según lo anterior, los museos son centros de aprendizajes de manera informal, donde la visita exhorta al alumno a un desarrollo integral y práctico, teniendo un escenario real para el desarrollo de la creatividad.

\section{Los museos y las tecnologías interactivas.}

Gracias al desarrollo de las nuevas tecnologías, los museos en este caso han ido evolucionando e incorporando nuevos contenidos con el objetivo de facilitar la transmisión del conocimiento. Esto a su vez permite desarrollar un mejor aprovechamiento de los elementos culturales existentes en cada uno de estos espacios, y que se genere un aprendizaje significativo.

Una de las características tecnológicas que se ha venido incorporando en sitios culturales como museos, centros de presentación del patrimonio, monumentos, entre otros, son las "tecnologías interactivas", esto debido a la experimentación en dichos espacios, en los cuales se consideró propicio el aumento de la relación entre usuarios y dispositivos o sistemas, tomando en cuenta la accesibilidad, que asegura su uso por los sectores de la población de países desarrollados, y que adquieran los conocimientos culturales.

Por otra parte, el concepto de la integración de la interactividad en ciertos espacios es y ha sido criticado por el resultado y las consecuencias que conlleva, esto a que algunos lo definen como ambientes de masificación, desorden y ruido, visualizado por museos en donde los usuarios normalmente hacen un uso desordenado de las herramientas.

En la mayoría de los casos se pretende integrar el uso de dichas tecnologías interactivas en los museos de manera instruida, es decir, dar indicaciones a los usuarios de cómo utilizar las herramientas. Sin embargo, la eficacia instructiva de la interactividad se encuentra limitada o nula, y en caso contrario tenemos la interacción sin instrucción, en donde encontramos la adquisición de conocimientos experimentales 
desencadenada por una conducta de acción-reacción. Se puede decir que ni el museo es un espacio necesariamente de enseñanza-aprendizaje, ni la interactividad facilita necesariamente un contexto instructivo de adquisición de conocimientos.

Por nombrar algún ejemplo de modelo interactivo que tuvo un impacto tanto en críticas como en el aprovechamiento de sus elementos, es el museo de ciencia "Exploratorium" en San Francisco, el cual explora la ciencia, arte y percepción humana, así mismo hace uso de 650 exhibiciones manuales e interactivas. En este espacio no se presenta ninguna colección artística, por lo cual no aspiraba a convertirse en un museo, sin embargo, la inclusión de dichas herramientas ya antes mencionadas lo colocan como uno de los primeros en utilizar estas tecnologías a finales del siglo $X X$.

\section{La Zona Arqueológica de Teotihuacán}

Teotihuacán, es una de las zonas arqueológicas más importantes de país, y en la antigüedad fue un centro religioso de gran importancia para la región del valle de Teotihuacán situado a $62.6 \mathrm{~km}$ del Zócalo de la Ciudad de México por carretera de acuerdo con la SCT (Secretaría de Comercio y Trasporte), la zona abarca una superficie de $21 \mathrm{~km}^{2}$, la obra de mayor importancia dentro de la zona es la Pirámide del Sol complementado con la Pirámide de la Luna además de un área conocida como la ciudadela entre las cuales esta los conjuntos habitacionales de Tepantitla, La Ventanilla, Tetitla, y Atetelco (Ortega, 2017).

La zona cuenta actualmente con distintos espacios además de las pirámides y estos son: Templo de Quetzalcóatl, un museo, un conjunto de superpuestos, el palacio de Quetzalpapálotl, la ciudadela, Centro de estudios teotihuacanos, Museo de los murales Teotihuacanos "Beatriz de la fuente y Plazas de la Calzada de los Muertos" (Ortega, 2017).

Gracias a todos estos atractivos que ha recibido y por la diversidad de servicios que ofrece se ha vuelto un atractivo de investigación para estudiante, pues los estudiantes representan una gran parte de la visita que recibe Teotihuacán anualmente. A continuación, se presenta la afluencia de estudiantes de estudiantes a la zona arqueológica mencionada en que va del año 2019.

\begin{tabular}{|c|c|c|c|c|c|c|c|}
\hline Mes & Eero & Fewren & Nazzo & Abril & Мapo & Junis & Total \\
\hline Nivel basico & 3,845 & 3,37 & 8,291 & 19,143 & 8,814 & 5271 & 40,187 \\
\hline Nwel Sysenor & 738 & 416 & 1,811 & 3,455 & 4,032 & 1783 & 8,665 \\
\hline
\end{tabular}

\section{Afluencia de estudiantes a la zona arqueológica de} Teotihuacan

Fuente: Elaboración propia con datos estadísticos de INAH

La zona ofrece a los turistas la grandeza de sus murales dentro de estos conjuntos habitacionales en la cual describe la cosmovisión y estilo de vida de sus antiguos habitantes. Acorde a distintos investigadores los mu rales que se encuentran en el sitio proporcionan información acerca de la cultura de los teotihuacanos, su religión exclusivamente para ser más específicos, pero en base a estas pinturas se puede generar una noción de como la religión influía dentro de la vida de los habitantes, en aspectos como sus cultos, rituales, deidades, y su cosmovisión.

Hasta la fecha no se sabe de forma certera cual es el origen esta cultura, así como la lengua que se habló ni el verdadero nombre de los habitantes fundadores de la ciudad. La información más cercana a estas incógnitas viene de las redacciones hechas en la época del virreinato, en las cuales se describe que Teotihuacán es un lugar mítico en el cual nació el quinto sol. La falta de documentos lleva a la dependencia e interpretación de los murales.

Por ello es que los murales cuentan con una gran importancia dentro de la zona, pero actualmente se ha sufrido una pérdida mayoritaria de murales que en los años 70's estaban en buenas condiciones y cada vez esto se vuelve más notorio.

A partir del año de 1978 la zona arqueológica fue declarada como patrimonio de la humanidad por la UNESCO, por cumplir con 6 de los 10 criterios de lista de patrimonios de la humanidad (Ortega, 2017).

\section{Resultados}

Para saber a cuántos alumnos de nivel superior se les aplicaría la encuesta se utilizó la siguiente fórmula para el cálculo de la población finita conocida, tomando en cuenta el número de alumnos con los que cuenta la plantilla estudiantil de la Escuela Superior de Tizayuca la cual es de 1497 alumnos y considerando un margen de error $5 \%$.

$$
n=\frac{Z_{s}^{2} \cdot N \cdot p \cdot q}{i^{2}(N-1)+Z_{*}^{2} \cdot p \cdot q}
$$

Donde:

$\mathrm{n}$ : Tamaño muestral

$\mathrm{N}$ : Tamaño de la población

$Z$ : Valor correspondiente a la distribu ción de Gauss, $Z_{\alpha=0.05}$ y $Z_{\alpha=0.01}=2.58$

$\mathrm{p}=$ Prevalencia esperada del parámetro a evaluar, en caso de desconocerse $(p=0.5)$, que hace mayor el tamaño muestral

$q: 1-p(S I p=30 \%, q=70 \%)$

$\mathrm{i}$ : Error que se prevé cometer si es del 10\%, i = 0.1

De acuerdo a los resultados arrojados por la formula se realizaron 73 encuestas de muestreo a alumnos del nivel medio superior de la Escuela superior de Tizayuca (ESTi- 
UAEH) los cuales rondan en una edad de 14 a 18 años de edad y el $54.7 \%$ son mujeres, donde la finalidad es identificar si los alumnos que han visitado la zona arqueológica de Teotihuacán, ha tenido un aprendizaje significativo y si proponen alguna tecnología interactiva.

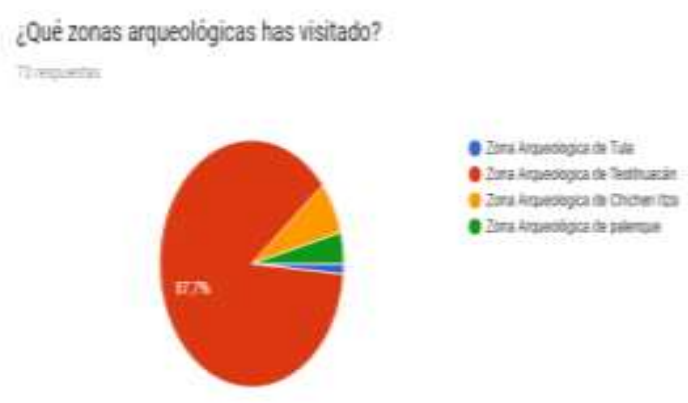

Gráfico 1

De acuerdo con la zona en la que se realiza la encuesta, los estudiantes tienen mayor preferencia por la zona arqueológica de Teotihuacán, seguido de Chichen Itzá, Palenque y Tula. Según el top 5 de las zonas arqueológicas más visitadas emitida por Segob Teotihuacán es la primera zona en ser visitada a nivel nacional por 1'859,818. Seguida por Chichen Itzá con 481,818. (Datatur, 2019).

¿Cuál ha sido el principal motivo de tus visitas?
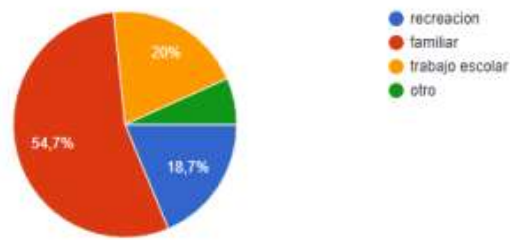

Gráfico 2

Dentro de los motivos de visita de los estudiantes, asistir a una zona arqueológica es más frecuente con la familia que incluso por algún motivo escolar o de interés propio.

\section{¿Cuál es el significado de la palabra "Teothuacán'?}

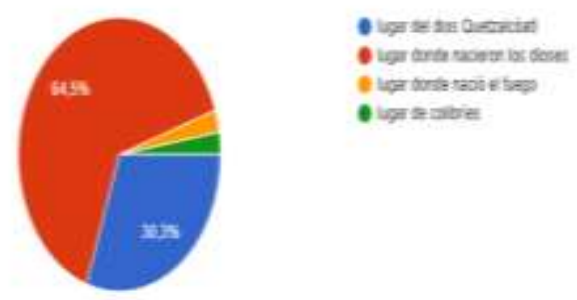

Gráfico 3
La mayoría de los estudiantes tienen el conocimiento básico de lo que fue Teotihuacán (64.5\%) y de su significado, así como de datos históricos del sitio.

\section{¿Sabes que civilización fundo esta zona?}
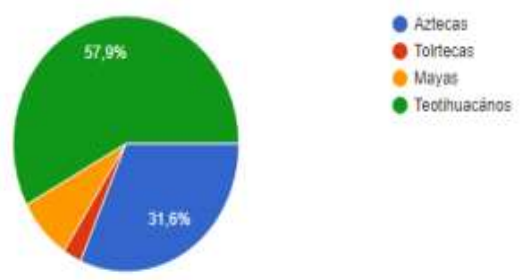

Gráfico 4

Más de la mitad de la muestra (57.9\%) tiene el conocimiento de que esta zona fue fundada por los teotihuacanos, sin embargo, existe un gran porcentaje de estudiantes que aún tienen la confusión de los fundadores de esta zona, confundiéndose con los aztecas.

\section{¿A qué dios se le ofrecian tributos en la pirámide el sol?}
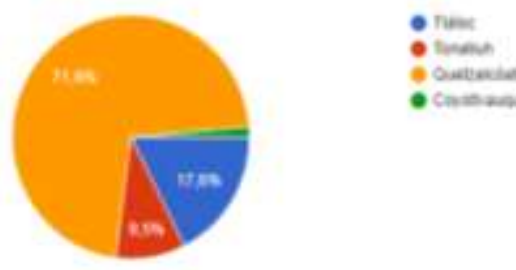

\section{Gráfico 5}

La mayoría de los estudiantes ( $71.6 \%$ )_consideran que la pirámide del sol es atribuida a Quetzalcóatl. Esto demuestra que pese a que muchos han visitado la zona arqueológica no han obtenido una información correcta, pues la pirámide es dedicada al dios de la lluvia "Tláloc", en lo cual, poco menos de la quinta parte conoce.

Se entiende que los alumnos tienen un conocimiento general en cuanto a Teotihuacán, sin embargo, la información que tiene del lugar no es detallada ya que muchos mostraron dudas en las respuestas. Esto indica que la información no es transmitida de manera correcta 


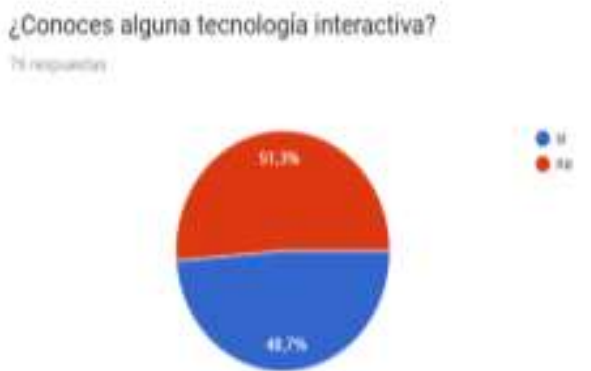

Gráfico 6

Pese al avance tecnológico que se está mostrando actualmente y al desarrollo innovador que existe en distintas áreas, aun no se logra difundir el uso de tecnologías interactivas como una herramienta cotidiana, pues poco más de la mitad (51.3\%) de la población sabe de dichas tecnologías.

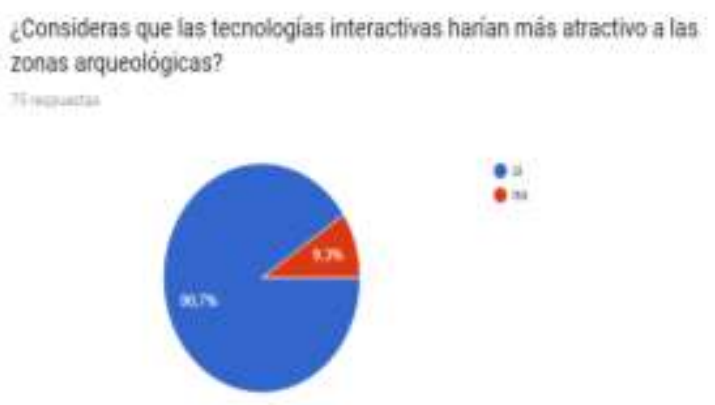

Gráfico 7

Los estudiantes encuestados reflejan algún interés por la inclusión de las tecnologías interactivas en este tipo de espacios culturales.

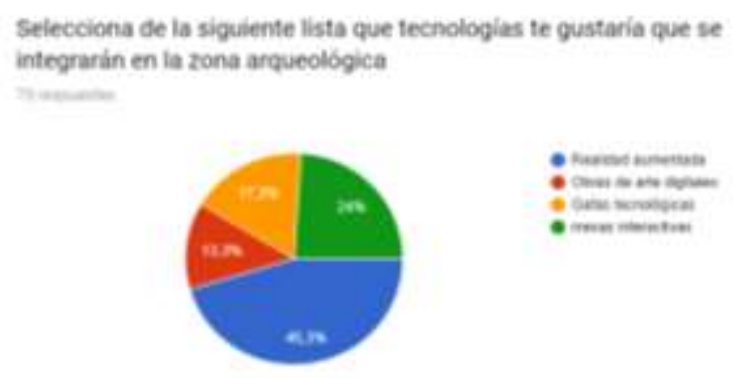

Gráfico 8

Se puede observar que el $45.3 \%$ de los encuestados eligieron la opción de realidad aumentada, el $24 \%$ mesas interactivas, El 17\% "gafas tecnológicas" y el 13.3\% eligió obras de arte digitales. Se optó por colocar en la encuesta estas cuatro opciones ya que son atractivas, de fácil implementación y representa una tendencia en los espacios culturales. Así mismo se puede definir que la tecnología más atractiva para los estudiantes de la escuela superior de Tizayuca (ESTI), es la realidad aumentada. Esto puede ser debido a que, por medio de esta herramienta, se consigue un modelo diferente de aprendizaje, en donde el resultado es la obtención de una mayor retención de información. Esto cumple con el principal objetivo de la interacción relación usuariotecnología.

\section{Conclusiones}

La Zona Arqueológica de Teotihuacán cuenta con un gran valor cultural e histórico, por ello se le ha otorgado el reconocimiento de "patrimonio de la humanidad", este reconocimiento también se les atribuye a lugares que suelen ser turismo de masas, debido al valor que tienen a nivel mundial. Teotihuacán al contar con una gran cantidad de visitantes al año y contar con una riqueza histórica es un sitio factible para la implementación de tecnologías interactivas que ayuden a nutrir y retener la información del sitio.

Esto se logra mediante la interacción entre los usuarios y la tecnología en donde el principal objetivo es modificar y crear diferentes procesos de enseñanza que satisfagan a distintos métodos de aprendizaje que tiene cada persona como lo son: aprendizaje procedimental, intelectual y físico de manera uniforme y asíse logre que el aprendizaje se retenga de una manera atractiva para los visitantes. Especialmente para estudiantes pues se les atribuye como unos de los principales visitantes que asisten al sitio ya sea por razones escolares o de índole familiar que son quienes cuentan con un interés por las tendencias tecnológicas.

Según los resultados se pudo observar que los alumnos que visitan la zona arqueológica no tienen un aprendizaje claro ni significativo de su historia, perdiéndose en cierto sentido el objetivo de lugar que consiste en propagar la cultura por medio de escenarios reales, por lo que las tecnologías interactivas muestran una alternativa para preservar el lugar, preservar la cultura e interesar a las nuevas generaciones de estudiantes.

Por ello las tecnologías interactivas en Teotihuacán son viables, pues Teotihuacán tiene grandes cantidades de visitantes anuales que asisten por motivos tanto culturales como de educación y estarán motivados por el uso de los medios digitales al ser un nuevo método de aprendizaje e investigación para estudiantes de nivel medio superior. 


\section{Referencias}

Bedolla, A. G. (17 de junio de 2015). Instituto Nacional de Antropologia e Historia. Recuperado el 10 de agosto de 2019, de Medios interactivos en museos deben potenciar su función educativa: https://inah.gob.mx/boletines/329-medios-interactivos-enmuseos-deben-potenciar-su-funcion-educativa

Cardona,H., Xavier, F., \& Campillo, R. (2009). Interactividad didactica en museos. redalyc,91-96. Obtenido de INTERACTIVIDAD DIDÁCTICA Y MUSEOS:

https://www.redalyc.org/pdf/3241/324127628010.pdf

Datatur. (2019). Datatur. Recuperado el 2 de Agosto de 2019, de llegadas a museos y zonas arqueologicas:

https://www.datatur.sectur.gob.mx/SitePages/ActividadesCultu rales.aspx

Espacio visual Europa.(13 de julio de 2017). Eve museo einnovacion . Obtenido de Museo y adapacion tecologica: https://evemuseografia.com/2017/07/13/museos-y-adaptaciontecnologica/

GAFA. (19 de Mayo de 2019). Obtenido de 5 Grandes museos interactivos del mundo: https://gafa.mx/museos-interactivos-mundo/

Garcia-Allen, J. (2019). Psicologia y Mente. Recuperado el 15 de agostto de 2019, de Psicologia y Mente: https://psicologiaymente.com/desarrollo/tipos-de-aprendizaje

Meritxell, M. (2002). Dialnet. Recuperado el 12 de Agosto de 2019, de interactividade interaccion:

file://C:/Users/BIBLIOTECA/Downloads/DialnetInteractividadEInteraccion-1252603.pdf

Ortega, C. V. (2017). El Patrimonio Arqueológico de Teotihuacán. Responsabilidad Social. Recupera do el 9 de agosto de 2019, de https://arqueologia mexicana.mx/mexico-antiguo/el-patrimonioarqueologico-de-teotihuacan-responsabilidad-social.

Red Nacionalde Información Cultural. (13 de Noviembre de 2018). Sistema de informacion cultural. Obtenido de Museo interactivo de tecnologia aplicada : https://sic.cultura.gob.mx/ficha.php?=mtableuseo\&table_id $=18$ 10

Vallejo, N. (28 de JULIO de 2015). ojulearning. Obtenido de 15 EJEMPLOS DE TECNOLOGIAS EN MÉXICO: https://ojulearning.es/2015/07/15-ejemplos-de-tecnologia-enmuseos/ 